\title{
Heterogeneidad en el involucramiento en acoso escolar: Un análisis de clases latentes
}

\author{
Heterogeneity in Involvement in School Bullying: A Latent Classes Analysis
}

Heterogeneidade no envolvimento do bullying escolar: Uma análise das classes latentes

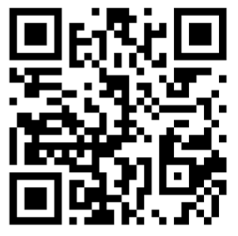

Carlos Mellado

Universidad Santo Tomás

Talca, Chile

carlosmellado@santotomas.cl

https://orcid.org/0000-0001-7894-9468

Pablo Méndez-Bustos

Universidad Católica del Maule

Talca, Chile

p.mendezb@gmail.com

https://orcid.org/0000-0002-9026-6974

Recibido • Received • Recebido: 10 / 09 / 2019

Corregido • Revised • Revisado: 19 / 05 / 2021

Aceptado • Accepted • Aprovado: 07 / 06 / 2021

\begin{abstract}
Resumen: El presente estudio tuvo como objetivo desarrollar una tipología de acoso escolar a través de una técnica estadística que minimiza el error de clasificación en los grupos. Esta tipología permite examinar cómo múltiples atributos del acoso escolar coexisten en las personas, sin separarlos artificialmente. Utilizando un análisis de clases latentes se modelaron seis ítems que representaban los roles de testigo, víctima y sujeto agresor en situaciones de acoso en 440 escolares de Chile, cuya edad fluctuó entre 14 y 20 años $(M=16,30$ y $D E=1,42)$. Se reportan dos modelos con cuatro y cinco clases respectivamente. En el primer modelo se observa un grupo denominado como "No involucrado" (14,5\%) el cual no ha participado en situaciones de acoso; un grupo denominado como "Testigo" (56,2\%) el cual solamente se ha involucrado como testigo en dichas situaciones; un grupo denominado como "Testigo y víctima" (13,3\%) el cual se ha implicado como testigo y víctima en situaciones de acoso y, finalmente, un grupo denominado como "Testigo, víctima y agresor" (16\%) el cual se ha implicado en todos los roles. En el modelo de cinco clases se observan los mismos grupos, más uno denominado como "Testigo y agresor" que se ha implicado como testigo y agresor en acoso escolar. Los resultados indican que la mayoría del estudiantado se ha involucrado en situaciones de acoso y que una parte importante se ha involucrado en más de un rol, lo que implica que la victimización y la perpetración del acoso no son mutuamente exclusivos.
\end{abstract}

Palabras claves: Acoso escolar; convivencia pacífica; violencia; tipología. 
http://doi.org/10.15359/ree.25-3.4

http://www.una.ac.cr/educare

educare@una.ac.cr

\begin{abstract}
The present study aimed to develop a typology of school bullying through a statistical technique that minimizes the classification error in groups. This typology allows examining how multiple attributes of bullying coexist in people without artificially separating those attributes. Using a latent class analysis, six items that represented the role of the witness, the victim, and the aggressor were modeled in school bullying situations in a sample of 440 Chilean schoolchildren between 14 and 20 years of age $(M=16,30$ and $S D=1,42)$. Two models are reported with four and five latent classes, respectively. In the four-class model, it can be seen a group called "Not Involved" (14,5\%) which has not been implicated in any of the roles of harassment; another group called "Witness" $(56,2 \%)$ which has only been involved as a witness in bullying situations; a group called "Witness and Victim" (13,3\%) which has been involved as a witness and victim in school bullying situations; and finally, a group called "Witness, Victim and Aggressor" (16\%) which has been involved in all roles. In the five-class model, the same groups are observed, plus one denominated as "Witness and Aggressor" implicated as a witness and aggressor in school bullying situations. The results show that most of the student body has been involved in bullying situations and that an important part has been involved in more than one role, which implies that victimization and perpetration of bullying are not mutually exclusive.
\end{abstract}

Keywords: Bullying, peaceful coexistence, violence, typology.

Resumo: O presente estudo teve como objetivo desenvolver uma tipologia de bullying através de uma técnica estatística que minimiza o erro de classificação nos grupos. Essa tipologia nos permite examinar como os múltiplos atributos do bullying coexistem nas pessoas sem separá-los artificialmente. Usando uma análise de classe latente, seis itens foram modelados representando os papéis de testemunha, vítima e sujeito agressor em situações de bullying em 440 estudantes chilenos entre 14 e 20 anos de idade $(M=16,30$ e $D E=1,42)$. Dois modelos com quatro e cinco classes latentes são relatados respectivamente. No modelo de quatro classes há um grupo chamado "Não envolvido" (14,5\%), que não esteve envolvido na participação de bullying, um grupo chamado "Testemunha" $(56,2 \%)$, que só esteve envolvido como testemunha em situações de bullying, um grupo chamado "Testemunha e vítima" $(13,3 \%)$ que esteve envolvido como testemunha e vítima em situações de assédio e finalmente um grupo chamado "Testemunha, vítima e agressor" que esteve envolvido em todas as funções. No modelo de cinco classes, os mesmos grupos são observados, e mais um chamado "Testemunha e agressor" (16\%), que esteve envolvido como testemunha e agressor no bullying. Os resultados indicam que a maioria dos estudantes já se envolveu em situações de bullying e que uma parte importante esteve envolvida em mais de uma função, o que implica que a vitimização e a perpetração de bullying não são mutuamente exclusivos.

Palavras-chave: Bullying, convivência pacífica, violência, tipologia.

\title{
Introducción
}

El acoso escolar o bullying fue conceptualizado a mediados de la década de 1980 por Olweus (1986) como la exposición de estudiantes a acciones negativas y repetitivas (i.e. intimidación, abuso, agresiones físicas o verbales) efectuadas por pares. En ese sentido, una característica 
http://doi.org/10.15359/ree.25-3.4

definitoria del acoso escolar es que existe un desequilibrio o asimetría de poder en desmedro de la víctima del acoso. Actualmente dicha definición no ha sufrido grandes cambios (Berger Silva, 2012; Hidalgo-Rasmussen et al., 2015; Olweus, 2013) aunque perspectivas ecológicas han puesto en relieve el rol que cumplen los testigos que validan el acoso (Berger y Rodkin, 2009), principalmente porque la reacciones y conductas de testigos pueden modificar el resultado de una situación de acoso (Barhight et al., 2013).

El fenómeno del acoso escolar se ha transformado en una preocupación constante para las personas investigadoras durante las dos últimas décadas (Berger Silva, 2012), ya que sistemáticamente ha sido relacionado con problemas emocionales y de ajuste tanto en víctimas como en sujetos acosadores (Nyulund-Gibson, Bellmore et al., 2007). El concepto víctima ha sido asociado con ansiedad social (Pavian y Vandebosch, 2016), síntomas depresivos (Cole et al., 2016) y problemas conductuales (Golmaryami et al., 2016). Además, ha sido relacionado con un mayor riesgo de conducta suicida (Klomek et al., 2008) y de muerte por accidente o lesiones autoinflingidas (Srabstein y Piaza, 2008). El concepto de sujeto acosador, en tanto, ha sido asociado con baja empatía y culpa (Fanti y Kimonis, 2012) y mayor ira y problemas conductuales (Golmaryami et al., 2016). La bibliografía ha mostrado que una significativa proporción de adolescentes ha sido víctima o ha perpetrado acoso escolar. El fenómeno de victimización (ser víctima de acoso) presenta proporciones de entre el 5,5\% y el 72\%, dependiendo de la población y el tipo de acoso escolar estudiado. La incidencia del acoso (cometer acoso), en tanto, muestra proporciones entre el 3\% y el 44\% (Pavian y Vandebosch, 2016). En Chile, el acoso escolar aumentó desde el 11\% en el año 2007 al 14,5\% en el año 2009 (Hidalgo-Rasmussen et al., 2015).

Cuando se estudia el acoso escolar una opción descriptiva-analítica es clasificar a las personas en subgrupos o tipos de víctimas y sujetos acosadores. En ese sentido, es necesario que los grupos conformados muestren, de manera precisa, las diferencias que existen entre las personas que conforman cada grupo (Nylund-Gibson, Bellmore et al., 2007). Para conformar los grupos, una opción es convertir una variable continua en una variable dicotómica, dividiendo la escala en algún punto de corte y asignando a los individuos con puntajes por sobre y por debajo de ese punto a grupos separados (MacCallum et al., 2002). El problema es que no existen guías claras para la elección de los puntos de cortes que separan los grupos, por lo mismo estos son elegidos arbitrariamente (Nyulund-Gibson, Bellmore et al., 2007; Solberg y Olweus, 2003). Por ejemplo, Berger Silva (2012) e Hidalgo-Rasmussen et al. (2015) categorizan a participantes de sus investigaciones como víctimas o no víctimas, considerando como criterio de corte una desviación estándar sobre el promedio del total de participantes en el puntaje de victimización. Ahora bien, el establecer puntos de corte presenta algunas falencias que pueden sesgar los resultados de las investigaciones que los utilizan. En primer lugar, no existen guías claras para la elección de los puntos de cortes que separan los grupos, por lo mismo estos son elegidos arbitrariamente (Nylund-Gibson, Bellmore et al., 2007; Soberg y Olweus 2003). El 
http://doi.org/10.15359/ree.25-3.4

http://www.una.ac.cr/educare

educare@una.ac.cr

cuestionamiento es el siguiente: ¿Cuál es el argumento sustantivo que permite catalogar como "víctima" a una persona con un puntaje por sobre una desviación estándar con respecto a la media en la variable acoso escolar? Si no existe dicho argumento, también se podría catalogar como víctimas, y así sucede actualmente en la investigación, a personas con puntajes por sobre 0,75 desviaciones estándar sobre la media (Graham et al., 2006) o a personas con puntajes por sobre 0,50 desviaciones estándar sobre la media (Juvonen et al., 2003). De esta manera, si la elección del punto de corte que define a una víctima de acoso escolar es arbitraria y depende de quien investiga, las consecuencias serán que una persona sea víctima de acoso o no, dependiendo de decisiones no necesariamente fundamentadas.

En segundo lugar, este método no entrega índices que cuantifiquen el error de clasificación de los sujetos en los grupos. Dicho error de clasificación finalmente resulta en una subestimación de las relaciones entre la variable acoso escolar y cualquier otra variable de interés (Bolck et al., 2004) y por lo mismo pueden aminorar la capacidad de las investigaciones para predecir diferencias entre los grupos de víctimas y no víctimas en su desarrollo psicosocial (Nylund-Gibson, Bellmore et al., 2007). Finalmente, la elección de puntos de corte arbitrarios impide comparar las prevalencias de acoso escolar que reportan distintos estudios (Soberg y Olweus 2003).

Ahora bien, el análisis de clases latentes (Latent Class Analysis, LCA) es un método estadístico que ha sido ampliamente utilizado en investigación para identificar subgrupos de personas o clases. Este análisis, más que confiar en predeterminados puntos de corte, asume que una variable categórica subyacente determina la pertenencia de personas en una clase o subgrupo latente en donde los distintos niveles de la variable latente corresponden a los distintos subgrupos o clases de personas. Dichas clases son exhaustivas y mutuamente excluyentes, y los individuos que las componen se caracterizan por presentar similares patrones de respuestas a ítems que corresponden a múltiples dimensiones de una variable (Bray et al., 2010).

En un modelo de clases latentes se estiman simultáneamente dos tipos de parámetros. La probabilidad de membrecía en la clase latente (latent class membership probabilities) representa la probabilidad de pertenecer a cada clase. La probabilidad condicional de respuesta al ítem (conditional item reponse probabilities), en tanto, describe la relación entre la pertenencia a la clase latente y las respuestas de una persona a los ítems en un momento específico en el tiempo (Cumsille et al., 2009), es decir, es la probabilidad de que la persona miembro de una clase responda a un ítem de una manera determinada.

Por ejemplo, Nylund-Gibson, Bellmore et al. (2007), a través de un LCA, modela tres clases o grupos de adolescentes según su sensación de victimización: un grupo que se considera "fuertemente" como víctima (FV), un grupo que se experimenta "levemente" como víctima (LV) y un grupo que no se considera como víctima (NV). Dicha investigación reporta que el grupo NV 
http://doi.org/10.15359/ree.25-3.4

muestra una probabilidad menor a 0,1 de responder afirmativamente a cada uno de los ítems que miden victimización (ser golpeado, ser víctima de chismes, etc.) mientras que el grupo FV, en tanto, muestra una probabilidad mayor a 0,75 de responder afirmativamente a los mismos ítems. Los sujetos participantes muestran una probabilidad de 0,2 de ser clasificados en el grupo FV, una probabilidad de 0,37 de ser clasificados en el grupo LV, y una probabilidad de 0,43 de ser clasificados en el grupo NV. Dichas probabilidades de clasificación pueden ser transformadas en porcentajes que indican la cantidad de personas que pertenecen a cada grupo.

Distintos estudios han utilizado esta aproximación para establecer clases o tipos de estudiantado involucrado en acoso escolar. Así, Lovegrove et al. (2012) describen cuatro clases que agrupan a adolescentes en USA y sus respectivos tamaños en porcentaje: un grupo de víctimas (15\%), uno de acosadores (13\%), uno de sujetos acosadores y víctimas a la vez (13\%) y finalmente un grupo de adolescentes que no se involucra en situaciones de acoso escolar (59\%). Chung y Lee (2020), utilizando la misma aproximación para clasificar a adolescentes de Corea, reportan un $7,1 \%$ de víctimas, un 10,1\% de sujetos acosadores, un $40,9 \%$ de sujetos acosadores y víctimas y finalmente un 31,9\% sin involucramiento. De esta forma, el análisis de clases latentes es una aproximación que puede ser utilizada para comparar prevalencias del acoso escolar en distintos países, sin utilizar puntos de corte arbitrarios para separar a víctimas de acoso.

De esta manera, el objetivo de la presente investigación es identificar sub-grupos de estudiantes que se involucran en situaciones de acoso escolar, a través de un análisis de clases latentes en una muestra de escolares de Chile, utilizando no solamente medidas del grado de victimización, según Nylund-Gibson, Bellmore et al. (2007), o de victimización y acoso como en Lovegrove et al. (2012) y Chung y Lee (2020), sino que también medidas del grado en que la muestra se involucra como testigo.

\section{Metodología}

\section{Participantes}

La muestra corresponde a 440 estudiantes de Chile que cursan entre $1^{\circ}$ y $4^{\circ}$ año de educación secundaria. Sus edades fluctúan entre 14 y 20 años $(M=16,30$ y $D E=1,42)$. El 54,7\% eran mujeres y el total de la muestra pertenece a un colegio municipal de la región del Maule. En relación con el nivel de ingresos familiar, el 50,9\% declara que su familia recibe 709 dólares al mes o menos. Finalmente, el $60 \%$ del alumnado reporta no haber repetido algún curso, el $25 \%$ declara consumir alcohol y el 88,8\% declara no consumir otro tipo de drogas (i.e. marihuana, cocaína, pasta base). 
http://doi.org/10.15359/ree.25-3.4

http://www.una.ac.cr/educare

educare@una.ac.cr

\section{Procedimiento}

Se contactó al colegio de donde se obtendría la muestra a través de su administración, se obtuvo el consentimiento para realizar la investigación en el establecimiento. Después se contactó a cada grupo de participantes en sus aulas, se les informó acerca de la investigación y se les invitó a colaborar, se les entregó una carta informativa y se les solicitó tanto su asentimiento para participar en la investigación como el consentimiento de sus familias. Para recolectar los datos se utilizaron cuestionarios de auto-reporte que fueron administrados a cada participante, en su propio colegio y salas de clases, por profesionales del área de ciencias sociales, con entrenamiento en la aplicación de pruebas. El procedimiento de administración del cuestionario no excedió de una hora.

El procedimiento de recolección de los datos respetó las normas éticas concordantes con la Declaración de Helsinki.

\section{Instrumentos}

Se utilizó el Cuestionario de Secundaria de Maltrato y Abuso de Poder (MIAP) adaptado para Chile por Lecannelier et al. (2011). Las dimensiones utilizadas por la presente investigación se denominan "testigo bullying" que evalúa la observación de prácticas reiterativas de acoso escolar, "victima bullying" que evalúa el grado en que estudiantes han sido víctima reiterada de acoso escolar y "agresor bullying" o frecuencia con que estudiantes han acosado a sus pares. Cada dimensión cuenta con una escala con formato de respuesta tipo Likert de cuatro puntos ( 1 = nunca, $2=$ a veces, $3=$ a menudo, $4=$ siempre $)$ en donde un mayor puntaje obtenido indica un mayor nivel en la dimensión evaluada. Para evaluar la consistencia interna de cada una de las escalas se realizó un análisis de alpha de Cronbach, y se encontró un $a=0,897$ para la escala testigo bullying, un $a=0,759$ para la escala victima bullying y un $a=0,745$ para la escala agresor bullying. Dichos coeficientes pueden ser considerados como aceptables.

Debido a las características del análisis estadístico utilizado en la presente investigación, se utilizaron dos ítems de la escala testigo ( $¡ H$ Has visto que insultan o colocan sobrenombres que ofendan y ridiculizan?), dos ítems de la escala víctima (¿Te han insultado o colocado sobrenombres que...?) y dos ítems de la escala agresor (¿Has insultado o has puesto sobrenombres que...?). Las respuestas a cada uno de los ítems fueron dicotomizadas, en donde las respuestas a veces, a menudo y siempre fueron colapsadas en una sola categoría que indicaba si el alumnado había sido testigo, víctima o agresor respectivamente en situaciones de acoso escolar. La respuesta nunca, en tanto, representó la situación inversa en que cada estudiante no había sido sujeto testigo, víctima o agresor en situaciones de acoso escolar. 


\section{Plan de análisis}

Se realizó un análisis de clases latentes (latent class analysis, LCA) para identificar heterogeneidad en los patrones de acoso escolar del alumnado. El enfoque utilizado para evaluar el ajuste del modelo en el análisis de clases latentes es el mismo que se usa en otros modelos con datos categóricos (Bray et al., 2010). La forma más efectiva para identificar el modelo óptimo es comparar el ajuste de una secuencia de modelos con diferente número de clases. Para comparar los modelos se utilizaron los índices AIC, BIC y BIC ajustado, donde el modelo que presenta indicadores con valores más bajos es aquel que presenta mejor ajuste. Es importante señalar que dichos índices se encuentran basados en el loglikelihood de los modelos ajustados y aplican diferentes penalizaciones tanto por el número de parámetros que presenta el modelo como por el tamaño de su muestra, por lo mismo es posible que cada índice de ajuste indique un modelo distinto como el mejor modelo (Nylund-Gibson, Asparouhov et al., 2007). De esta manera, también se utilizaron la prueba de Lo-Mendel-Rubin (LMR) y la prueba BLRT (Bootstrap Likelihood Ratio Test), dichas pruebas comparan la mejora en el ajuste entre modelos con clases colindantes (i.e. compara el modelo que presenta $k$ clases con el modelo que presenta $k-1$ clases) y entrega un valor $p$ que puede ser utilizado para determinar si hay una mejora estadísticamente significativa en el ajuste cuando se incluye una clase más. Finalmente se consideró la interpretabilidad del modelo, es decir, que las probabilidades de respuesta al ítem distinguieran cada clase de las otras, que ninguna clase tuviera una probabilidad de membrecía cercana a cero y que fuera posible asignar una etiqueta con significado a cada clase (Lanza et al., 2007).

\section{Resultados}

Modelos con dos, tres, cuatro y cinco clases fueron comparados para identificar el número de clases que entrega un adecuado balance entre ajuste y parsimonia, cumpliendo con el criterio de interpretabilidad. En la Tabla 1 se reportan el BIC, el AIC, el LMR y el BLRT de cada uno de los modelos de acoso escolar. Se puede observar que el valor $p$ del LMR del modelo de cinco clases no fue significativo, lo que indica que dicho modelo no presenta un ajuste significativamente mejor que el modelo de cuatro clases. El menor AIC lo presenta el modelo de cinco clases y el menor BIC lo obtuvo el modelo con cuatro clases. Dado que los índices de ajuste no identificaron unilateralmente un modelo único como correcto y que el presente reporte tiene como objetivo describir la técnica del LCA para distinguir tipos de escolares involucrados en acoso escolar, no se elegirá un modelo único como correcto y se reportarán tanto los parámetros del modelo con cuatro clases como aquellos del modelo con cinco clases. La Tabla 1 también reporta la entropía del modelo de cuatro y cinco clases, en donde valores cercanos a uno indican una clara separación de las clases entre sí (Celeux y Soromenho, 1996). En ese sentido, el modelo de cuatro clases presenta una mayor separación, es decir, las clases se diferencian mejor unas de otras. 
http://doi.org/10.15359/ree.25-3.4

http://www.una.ac.cr/educare

educare@una.ac.cr

Tabla 1: Comparación de modelos de acoso escolar

\begin{tabular}{lrrllll}
\hline Número de Clases & AIC & BIC & BIC Ajustado & $\begin{array}{c}\text { LMR } \\
\text { Valor } p\end{array}$ & $\begin{array}{c}\text { BLRT } \\
\text { Valor } p\end{array}$ & Entropía \\
\hline 2 & 2402,20 & 2455,03 & 2413,78 & $<, 001$ & $<, 001$ & 0,730 \\
3 & 2313,56 & 2394,83 & 2331,36 & $<, 001$ & $<, 001$ & 0,818 \\
4 & 2290,48 & $\mathbf{2 4 0 0 , 2 1}$ & $\mathbf{2 3 1 4 , 5 2}$ & $<, 001$ & $<, 001$ & 0,947 \\
5 & $\mathbf{2 2 8 5 , 2 1}$ & 2423,38 & 2315,48 &, 124 & $<, 001$ & 0,858 \\
\hline
\end{tabular}

$\mathrm{AIC}=$ Akaike Information Criterion; $\mathrm{BIC}=$ Bayesian Information Criterion; $\mathrm{LMR}=$ Lo-Mendell-Rubin Test; $\mathrm{BLRT}=$ Bootstrap Likelihood Ratio Test. En negrita el menor AIC, BIC y BIC Ajustado.

Nota: Elaboración propia.

Las probabilidades condicionales de respuesta a cada ítem para el modelo de cuatro y cinco clases se reportan en la Tabla 2. Dichos parámetros representan la probabilidad de decir "si" (i.e. ser sujeto testigo, víctima o agresor en situaciones de acoso) a cada ítem, condicional a la pertenencia a cada clase. Cuanto más se aleja una probabilidad del,50 más representativo es el ítem de la clase, y todo valor cercano a ,50 indica que la respuesta a ese ítem no se relaciona con esa clase.

Los patrones de respuesta observados para el modelo de cuatro clases sugieren que emergió una clase etiquetada como "Sujeto no involucrado", en donde las probabilidades de respuesta positiva a los ítems fueron iguales o inferiores a ,03 y, por lo tanto, cada estudiante que se clasifica en ella muestra una muy baja probabilidad de haber sido testigo, víctima o sujeto agresor en situaciones de bullying. Una clase etiquetada como "testigo", donde las probabilidades de respuesta positiva a los ítems de observación de situaciones de acoso fueron iguales o superiores a , 81 y las probabilidades de respuesta positiva al resto de los ítems fueron todas menores a , 13 y, por lo tanto, cada estudiante en ella muestra alta probabilidad de haber sido testigo de situaciones de acoso y baja probabilidad de ser víctima o sujeto agresor. También se encontró una clase etiquetada como "testigo y víctima", en donde las probabilidades de respuesta positiva a los ítems de observación y vivencia de situaciones de acoso fueron superiores a ,84 (excepto el ítem víctima: sobrenombres) y las probabilidades de respuesta positiva a los ítems de agresión fueron menores a ,40; por lo tanto, cada adolescente en ella muestra alta probabilidad de haber sido testigo y víctima de situaciones de acoso, pero una baja probabilidad de ser sujeto agresor. La cuarta clase que surgió fue etiquetada como "testigo, víctima y sujeto agresor", en donde las probabilidades de respuesta positiva a todos los ítems fueron superiores a ,70 y, por lo tanto, cada escolar que se clasifica en ella muestra alta probabilidad de haber sido testigo, víctima y sujeto agresor en situaciones de acoso escolar. 
http://doi.org/10.15359/ree.25-3.4

En el modelo de cuatro clases la clase "testigo" fue la más común $(56,2 \%)$, seguida de la clase "testigo, víctima y sujeto agresor", (16\%) y la clase "sujeto no involucrado" (14,5\%). La clase menos común, en tanto fue "testigo y víctima" (13,3\%).

Tabla 2: Probabilidades de respuesta al ítem del acoso escolar condicional a la membrecía a la clase (modelos de cuatro y cinco clases)

\begin{tabular}{|c|c|c|c|c|c|c|c|}
\hline \multirow[b]{2}{*}{ Clase latente } & \multicolumn{7}{|c|}{ Modelo de cuatro clases } \\
\hline & $\begin{array}{c}\text { Testigo: } \\
\text { Sobrenombres }\end{array}$ & $\begin{array}{l}\text { Testigo: } \\
\text { Molestar }\end{array}$ & $\begin{array}{c}\text { Víctima: } \\
\text { Sobrenombres }\end{array}$ & $\begin{array}{l}\text { Víctima: } \\
\text { Molestar }\end{array}$ & $\begin{array}{c}\text { Agresor: } \\
\text { Sobrenombres }\end{array}$ & $\begin{array}{l}\text { Agresor: } \\
\text { Molestar }\end{array}$ & $\begin{array}{l}\text { Probabilidad de } \\
\text { membrecía }\end{array}$ \\
\hline $\begin{array}{l}\text { Sujeto } \\
\text { Involucrado }\end{array}$ & ,001 & ,001 &, 001 &, 036 &, 013 & ,010 & , 145 \\
\hline Testigo &, 817 & ,921 &, 060 &, 001 & ,067 & 130 &, 562 \\
\hline Testigo y víctima &, 845 & ,999 & ,475 & ,999 & ,001 & 395 & 133 \\
\hline \multirow[t]{2}{*}{$\begin{array}{l}\text { Testigo, víctima } \\
\text { y agresor }\end{array}$} & 955 & ,970 & ,703 &, 580 & ,999 & 792 & 160 \\
\hline & \multicolumn{7}{|c|}{ Modelo de cinco clases } \\
\hline $\begin{array}{l}\text { Sujeto } \\
\text { No involucrado }\end{array}$ & ,001 & 174 & ,001 & ,033 & ,017 & ,015 & 170 \\
\hline Testigo & 883 & 908 & ,072 & ,001 &, 001 & ,086 & 456 \\
\hline Testigo y agresor &, 814 & ,999 & ,001 &, 001 & ,608 &, 559 & 109 \\
\hline Testigo y víctima &, 852 & ,999 & ,476 & ,999 & ,037 & 396 & , 139 \\
\hline $\begin{array}{l}\text { Testigo, víctima } \\
\text { y agresor }\end{array}$ & ,962 & ,962 &, 893 & ,700 & ,999 & ,775 & 124 \\
\hline
\end{tabular}

Nota: Elaboración propia.

Los patrones de respuesta observados para el modelo de cinco clases indican que surgieron las clases de sujetos "no involucrado", "testigo", "testigo y víctima" y "testigo, víctima y agresor", las cuales tienen significados análogos a las detectadas en el modelo de cuatro clases. Además, surgió una quinta clase que fue etiquetada como sujeto "testigo y agresor" ", en donde las probabilidades de respuesta positiva a los ítems de observación de situaciones de acoso fueron superiores a ,81; las probabilidades de respuesta positiva a los ítems de agresión fueron 
http://doi.org/10.15359/ree.25-3.4

http://www.una.ac.cr/educare

educare@una.ac.cr

superiores a ,55 y las probabilidades de respuesta positiva a los ítems de victimización fueron inferiores a ,001; por lo tanto, cada adolescente que se clasifica en ella muestra alta probabilidad de haber sido sujeto testigo y agresor en situaciones de acoso, pero una baja probabilidad de ser víctima. En este modelo, la clase "testigo" fue la más común $(45,6 \%)$, seguida de las clases "no involucrado" (17\%) y "testigo y víctima" (13,9\%). Las clases menos comunes, fueron las de "testigo, víctima y agresor", (12,4\%) y testigo y víctima (10,9\%).

\section{Discusión}

Los resultados de la presente investigación indican que la mayoría del alumnado de la muestra se ha visto involucrado en situaciones de acoso, ya sea en el rol de testigo, víctimas o agresor; se concluye, además, que una parte importante de escolares se ha involucrado en más de un rol (e.g. escolares que reportan ser sujetos testigos, víctimas y agresores). Dichos resultados son consistentes con investigaciones anteriores que muestran una alta prevalencia del fenómeno (Pavian y Vandebosch, 2016), una alta presencia de testigos de acoso (Barhight et al., 2013) y la existencia de escolares que se han involucrado tanto en el rol de víctima como de sujeto agresor (Juvonen et al., 2003).

La evidencia es escasa y son pocos los estudios respecto a la incidencia del acoso en Chile o el porcentaje del alumnado que se ha involucrado en cada uno de sus roles. En el año 2018, la Encuesta Nacional de la Juventud (Instituto Nacional de la Juventud [INJUV], Ministerio de Desarrollo Social y Familia, 2018) informó que un 29,3 \% de estudiantes de Chile entre 15-19 años fueron víctimas de acoso, alguna vez en su vida, en su lugar de estudio. Por otra parte, Herrera-López et al. (2018), después de realizar una revisión bibliográfica de acoso escolar en Latinoamérica, con el fin de comparar las incidencias del fenómeno entre países, señalan que solamente tres estudios analizan la incidencia global del acoso escolar en Chile; concluyen que este indicador se encuentra en torno al 45\%. Herrera-López et al. (2018) también señalan que solamente dos estudios chilenos indican el rol que el estudiantado cumple en un contexto de acoso escolar, indican que este indicador se encuentra cercano al 10\% tanto en el caso de sujetos acosadores como víctimas. Garaigordobil Landazabal et al. (2018), en un estudio similar al anterior, encuentran solamente un estudio chileno que entrega información acerca de la frecuencia en que el alumnado se involucra en cada uno de los roles del acoso escolar, indican que hay un $25 \%$ de víctimas, un $14,2 \%$ de sujetos agresores y un $11,5 \%$ de estudiantes que han cumplido ambos roles. En cualquier caso, los resultados del presente estudio concuerdan con las incidencias entregadas por las investigaciones realizadas con muestras chilenas presentadas en esas revisiones bibliográficas.

Ahora bien, a diferencia de los estudios anteriores, el enfoque analítico usado en la presente investigación permite describir los distintos subgrupos de individuos a través de una configuración resultante de la conjugación de múltiples dimensiones de una variable (von Eye y 
http://doi.org/10.15359/ree.25-3.4

Bogart, 2006). Así, dicho enfoque permite describir diversas actitudes y conductas relacionados con el acoso escolar en distintos subgrupos de la población (Bergman y El-Khouri, 2003; Heberle et al., 2015), y establecen perfiles que pueden ser útiles en la elaboración de estrategias tanto de prevención como intervención. Esto último es particularmente importante, ya que el bienestar y la salud mental en etapas tempranas de la vida influye en las proyecciones futuras de las personas (Organización Mundial de la Salud [OMS], 2003).

En ese sentido, entre los perfiles encontrados en esta investigación destaca aquel que conjuga los tres posibles roles que una persona puede asumir en una situación de acoso: testigo, víctima y sujeto agresor, el cual no ha sido descrito en investigaciones previas realizadas en Chile con metodologías más tradicionales (Herrera López et al., 2018). Este resultado confirma lo planteado originalmente por Olweus (1986), quien indica que la perpetración del acoso y la victimización no son mutuamente exclusivas y que, en ciertos grupos de jóvenes, esos roles tienden a superponerse. Además, este perfil es particularmente crítico, ya que según Shao et al. (2014), presenta un ajuste social y emocional mucho más bajo que cuando solamente se es víctima o sujeto agresor (i.e. mayores indicadores de depresión, ansiedad y soledad y menores indicadores de logro escolar). Por lo mismo, se hace importante identificarlo adecuadamente para generar estrategias de intervención focalizadas, ya que también se ha señalado que este grupo es menos apoyado por docentes y más desaprobado y agredido por sus pares (Shao et al. (2014).

Ahora bien, diferentes investigaciones han intentado estudiar el acoso escolar estableciendo clasificaciones que permitan entender estas relaciones de violencia (Haynie et al., 2001; Valadez et al., 2011). La importancia de una adecuada clasificación radica en la posibilidad de contribuir en un abordaje más eficiente de esta problemática, que mejore los modelos explicativos y favorezca el desarrollo de nuevas estrategias de intervención. El LCA permite cumplir cabalmente con ese objetivo, ya que es una técnica estadística que identifica grupos de personas que funcionan de manera similar en relación con un fenómeno como el acoso escolar, pero que, a la vez, funcionan diferenciadamente cuando se les compara con otros subgrupos de personas. Además, dicha técnica ofrece índices de ajuste del modelo estadístico testeado (i.e. modelos con distintos números de clases o subgrupos) que ayudan a la elección del modelo que mejor ajusta a los datos e incorpora una estimación del error de clasificación del estudiantado en los grupos. Si bien, la utilización del LCA en el estudio del acoso escolar implica ciertos requisitos en cuanto al tamaño muestral (se necesitan muestras "grandes") y a un software estadístico especializado para estimar los parámetros del modelo (e.g. Mplus) dichas limitaciones se compensan ampliamente con las ventajas que entrega la utilización de la técnica.

Finalmente, la presente investigación presenta ciertas limitaciones que es importante puntualizar. Primero, la información acerca del acoso escolar que se utilizó para modelar las clases fue obtenida a través del auto-reporte adolescente y no a través de observaciones directas de las interacciones entre el alumnado, por lo tanto, se recabó información acerca 
http://doi.org/10.15359/ree.25-3.4

http://www.una.ac.cr/educare

educare@una.ac.cr

de la percepción de involucramiento en situaciones de acoso. Además, dado que los ítems utilizados en la presente investigación no son exhaustivos, tampoco se puede descartar que otras dimensiones del acoso escolar (e.g. involucramiento en agresiones físicas, etc.) puedan ayudar a modelar las clases. Segundo, no se estimó la relación entre las clases de acoso escolar y otras variables que, según la bibliografía, inciden en la victimización o en la agresión. De esta manera, futuras investigaciones podrían ingresar al modelo predictores de la probabilidad de clasificación de los sujetos en cada uno de los grupos o clases.

\section{Declaración de Material complementario}

Este artículo tiene disponible, como material complementario:

-La versión preprint del artículo en https://doi.org/10.5281/zenodo.3923038

\section{Referencias}

Barhight, L. R., Hubbard, J. A. y Hyde, C. T. (2013). Children's physiological and emotional reactions to witnessing bullying predict bystander intervention. Child Development, 84(1), 375-390. https://doi.org/10.1111/j.1467-8624.2012.01839.x

Berger Silva, C. (2012). Trayectorias de victimización escolar: Características y factores de riesgo en adolescentes chilenos. Universitas Psychologica, 11(1), 103-118. https://doi.org/10.11144/ Javeriana.upsy11-1.tvec

Berger, C. y Rodkin, P. C. (2009). Male and female victims of male bullies: Social status differences by gender and informant source. Sex Roles, 61(1-2), 72-84. https://doi.org/10.1007/ s11199-009-9605-9

Bergman, L. R. y El-Khouri, B. M. (2003). A person-oriented approach: Methods for today and methods for tomorrow. New Directions for Child \& Adolescent Development, (101), 25-38. https://doi.org/10.1002/cd.80

Bolck, A., Croon, M. y Hagenaars, J. (2004). Estimating latent structure models with categorical variables: One-step versus three-step estimators. Political Analysis, 12(1), 3-27. https://doi. org/10.1093/pan/mph001

Bray, B. C., Lanza, S. T. y Collins, L. M. (2010). Modeling relations among discrete developmental processes: A general approach to associative latent transition analysis. Structural Equation Modeling: A Multidisciplinary Journal, 17(4), 541-569. https://doi.org/10.1080/10705511.20 $\underline{10.510043}$

Celeux, G. y Soromenho, G. (1996). An entropy criterion for assessing the number of clusters in a mixture model. Journal of Classification, 13(2), 195-212. https://doi.org/10.1007/ $\underline{\text { BF01246098 }}$ 
http://doi.org/10.15359/ree.25-3.4

Chung, J. Y. y Lee, S. (2020). Are bully-victims homogeneous? Latent class analysis on school bullying. Children and Youth Services Review, 112(2). https://doi.org/10.1016/j. childyouth.2020.104922

Cole, D. A., Zelkowitz, R. L., Nick, E., Martin, N. C., Roeder, K. M., Sinclair-McBride, K. y Spinelli. T. (2016). Longitudinal and incremental relation of cybervictimization to negative selfcognitions and depressive symptoms in young adolescents. Journal of Abnormal Child Psychology, 44(7), 1321-1332. https://doi.org/10.1007/s10802-015-0123-7

Cumsille, P., Darling, N., Flaherty, B. y Martínez, M. L. (2009). Heterogeneity and change in the patterning of adolescents' perceptions of the legitimacy parental authority: A latent transitional model. Child Development, 80(2),418-432. https://doi.org/10.1111/j.14678624.2009.01269.x

Fanti, K. A. y Kimonis, E. R. (2012), Bullying and victimization: The role of conduct problems and psychopathic traits. Journal of Research on Adolescence, 22(4), 617-631. https://doi. org/10.1111/j.1532-7795.2012.00809.x

Graham, S., Bellmore, A. D. y Mize, J. (2006). Peer victimization, aggression, and their cooccurrence in middle school. Pathways to adjustment problem. Journal of Abnormal Child Psychology, 34(3), 363-378. https://doi.org/10.1007/s10802-006-9030-2

Golmaryami, F. N., Frick, P. J., Hemphill, S. A., Kahn, R. E., Crapanzano, A. M. y Terranova, A. M. (2016). The social, behavioral, and emotional correlates of bullying and victimization in a school-based sample. Journal of Abnormal Child Psychology, 44(2), 381-391. https://doi. org/10.1007/s10802-015-9994-x

Garaigordobil Landazabal, M., Mollo-Torrico, J. P. y Larrain Mariño, E. (2018). Prevalencia de bullying y cyberbullying en Latinoamérica: Una revisión. Revista Iberoamericana de Psicología, 11(3), 1-18. https://doi.org/10.33881/2027-1786.rip.11301

Heberle, A. E., Briggs-Gowans, M. J. y Carter, A. S. (2015). A person-oriented approach to identifying parenting styles in mothers of early school-age children. Infant and Child Development, 24(2), 130-156. https://doi.org/10.1002/icd.1888

Haynie, D. L., Nansel, T., Eitel, P., Crump, A. D., Saylor, K., Yu, K. y Simons-Morton, B. (2001). Bullies, victims and bullies/victims: Distinct group of at-risk youth. Journal of Early Adolescence, 21(1), 29-49. https://doi.org/10.1177/0272431601021001002

Herrera-López, M., Romera, E.M.y Ortega-Ruiz, R.(2018). Bullying y cyberbullying en Latinoamérica: Un estudio bibliométrico. Revista Mexicana de Investigación Educativa, 23(76), 125-155. https://www.comie.org.mx/revista/v2018/rmie/index.php/nrmie/article/view/1142/1130 
http://doi.org/10.15359/ree.25-3.4

http://www.una.ac.cr/educare

educare@una.ac.cr

Hidalgo-Rasmussen, C., Molina, T., Molina, R., Sepúlveda, R., Martínez, V., Montaño,R., González, E. y George, M. (2015). Bullying y calidad de vida relacionada con la salud en adolescentes escolares chilenos. Revista Médica de Chile, 143(6), 716-723. https://doi.org/10.4067/S003498872015000600004

Instituto Nacional de la Juventud, Ministerio de Desarrollo Social y Familia. (2018). 9 Encuesta nacional de juventud. Autor. https://www.injuv.gob.cl/sites/default/files/ix encuesta nacional de la juventud 2018.pdf

Juvonen, J., Graham, S. y Schuster, M. A. (2003). Bullying among young adolescents: The strong, the weak, and the troubled. Pediatrics, 112(6 PT), 1231-1237. https://doi.org/10.1542/ peds.112.6.1231

Klomek, A. B., Marrocco, F., Kleinman, M., Schonfeld, I. S. y Gould, M. S. (2008). Peer victimization, depression, and suicidality in adolescents. Suicide and Life-Threatening Behavior, 38(2), 166-180. https://doi.org/10.1521/suli.2008.38.2.166

Lanza, S. T., Collins, L. M., Lemmon, D. R. y Schafer, J. L. (2007). PROC LCA: A SAS procedure for latent class analysis. Structural Equation Modeling, 14(4), 671-694. https://doi. org/10.1080/10705510701575602

Lecannelier, F., Varela, J., Rodríguez, J., Hoffman, M., Flores, F. y Ascanio L. (2011). Validación del Cuestionario de Maltrato entre Iguales por Abuso de Poder (MIAP) para escolares. Revista Médica de Chile, 139(4), 474-479. https://doi.org/10.4067/\$0034-98872011000400009

Lovegrove, P. J., Henry, K. L. y Slater, M. D. (2012). Examination of the predictors of latent class typologies of bullying involvement among middle school students. Journal of School Violence, 11(1), 75-93. https://doi.org/10.1080/15388220.2011.631447

MacCallum, R. C., Zhang, S., Preacher, K. J.y Rucker, D. D. (2002). On the practice of dichotomization of quantitative variables. Psychological Methods, 7(1), 19-40. https://doi.org/10.1037//1082989X.7.1.19

Nylund-Gibson, K. L., Asparouhov, T. y Muthén, B. O. (2007). Deciding on the number of classes in latent class analysis and growth mixture modeling: A monte carlo simulation study. Structural Equation Modeling, 14(4), 535-569. https://doi.org/10.1080/10705510701575396

Nylund-Gibson, K., Bellmore, A., Nishina, A. y Graham, S. (2007). Subtypes, severity, and structural stability of peer victimization: What does latent class analysis say? Child Development, 78(6), 1706-1722. https://doi.org/10.1111/j.1467-8624.2007.01097.x

Olweus, D. A. (1986). The Olweus Bully/victim questionnaire [Mimeo]. Research Center for Health Promotion, University of Bergen. 
http://doi.org/10.15359/ree.25-3.4

OlweusD.A.(2013).School bullying:Developmentand some importantchallenges. AnnualReview of Clinical Psychology, 9, 751-780. https://doi.org/10.1146/annurev-clinpsy-050212-185516

Organización Mundial de la Salud. (2003). Informe mundial sobre la salud 2003: Forjemos el futuro. Autor. http://www.who.int/whr/2003/en/whr03 es.pdf

Pavian, S. y Vandebosch, H. (2016). An investigation of short-term longitudinal associations between social anxiety and victimization and perpetration of traditional bullying and cyberbullying. Journal of Youth and Adolescence, 45(2), 328-339. https://doi.org/10.1007/ s10964-015-0259-3

Shao, A., Liang, L., Yuan, C. y Bian, Y. (2014). A latent class analysis of bullies, victims and aggressive victims in chinese adolescence: Relations with social and school adjustments. PLOS ONE, 9(4), 1-8. https://doi.org/10.1371/journal.pone.0095290

Solberg, M. E. y Olweus, D. (2003). Prevalence estimation of school bullying with the olweus bully/victim questionnaire. Aggressive Behavior, 29(3), 239-268. https://doi.org/10.1002/ ab.10047

Srabstein J. y Piazza, T. (2008). Public health, safety and educational risks associated with bullying behaviors in American adolescents. International Journal of Adolescent Medicine and Health, 20(2), 223-233. https://doi.org/10.1515/IJAMH.2008.20.2.223

Valadez, I., Amezcua, R., González, N., Montes, R. y Vargas, V. (2011). Maltrato entre iguales e intento suicida en sujetos adolescentes escolarizados. Revista Latinoamericana de Ciencias Sociales, Niñez y Juventud, 9(2), 783-796. http://revistaumanizales.cinde.org.co/rlcsnj/ index.php/Revista-Latinoamericana/article/view/473/265

von Eye, A. y Bogat A. G. (2006). Person-oriented and variable-oriented research: Concepts, results, and development. Merrill-PalmerQuarterly, 52(3), 390-420. https://doi.org/10.1353/ $\underline{\text { mpq.2006.0032 }}$ 